\title{
The Location Technology Research on Single Pulse Active Location Cooperate with Passive Location
}

\author{
Bing Sun ${ }^{1, a}$, Jingqing $\mathrm{LuO}^{2, \mathrm{~b}}$, ShiLong $\mathrm{Wu}^{3}$, Youjun $\mathrm{Liu}^{4}$ \\ ${ }^{1}$ Electronic Engineering Institute, Hefei, 230037, China \\ ${ }^{2}$ Electronic Engineering Institute, Hefei, 230037, China \\ ${ }^{3}$ Electronic Engineering Institute, Hefei, 230037, China \\ ${ }^{4}$ Electronic Engineering Institute, Hefei, 230037, China \\ aemail: 13721052122@163.com, bemail:18156065046@126.com
}

Keywords: Comprehensive Location; Single Pulse; Location Accuracy

\begin{abstract}
The technology of combining single pulse active location with passive location, the question of accomplishing accurate target location as well as avoiding the problem that active detection signals are easy to be intercepted and identified need more research. The location performance and error distribution features of passive detection of remote target are analyzed in the issue at first. Then the location performance and error distribution features of single pulse active detection is put forward. In the end, the location system of single pulse active location cooperate with passive location is proposed. At the same time, the maximum likelihood estimation location result of the system is deduced and the location performance is analyzed. With the theoretical analysis and simulation approach proposed, single pulse active location system have high radial location accuracy, and the passive location system have high tangential location accuracy. Comprehensive location can have high radial location accuracy as well as high tangential location accuracy.
\end{abstract}

\section{Introduction}

The enemy with high-value and advanced combat platform may often met in the air defense, early detection and accurately location of the enemy combat platform play an important role in the battle.

The early detection generally need the launch platform, and the method of passive location can instantaneously detect radiation source within line-of-sight. For the passive detection of radiation source, the single combat is difficult to meet demand while the multi-machine coordination is a common combat form in general.

General active detection is easy to be intercepted and identified, as well as facing with the comprehensive electronic jamming and the threat of anti-radiation weapon attack. The electromagnetic signals aren't emitted by the multi-machine synergetic passive location system itself, thus it can't be discovered by the enemy with its advantages of strong concealment. But for the remote targets from enemy incoming direction, the multi-machine synergetic passive location can only obtain a higher angle location accuracy (tangential location accuracy) [1-5], and a poorer distance location accuracy (radial location accuracy). In order to make the system gain higher location accuracy and avoid the enemy find our detection intentions, thus let the system with low detection performance, the host machine can carry out active detection through taking instantaneous mode to emit single pulse. The method can avoid the problem that general active detection signals are easy to be intercepted and identified, as well as acquire the higher distance location accuracy. In this mode, the launch direction is basically guided by passive detection signals, can achieve better range with low accuracy. So it can combine the angle information of multi-machine synergetic passive location to gain higher location accuracy[6-9].

The remote location features and the error distribution features of multi-machine synergetic 
passive detection are analyzed in the issue at first. Then the location performance of single pulse active detection is proposed. In the end, the maximum likelihood estimation location result of the system is deduced as well as the location performance is analyzed by combining the location information of single pulse active detection with synergetic passive detection.

\section{Remote Target Synergetic Passive Location}

In order to obtain high positioning accuracy, multi-machine synergy often uses TDOA system for remote radiation source targets.

TDOA is also called hyperbolic location, which locate the radiation source targets by means of dealing the difference between the signal arrival time that collected by three or more observatory. In two-dimensional plane, the difference between the radiation source signal arrival time determine a pair of hyperbola with two station for focuses, using three station to form two pairs of hyperbolic and generate intersection, recycling direction finding information to eliminate the false points, which can confirm the position of the radiation source.

Supposing that the system is composed of one host machine and two wing machines, the position of the target to be measured is $(x, y)$, Assuming that the host machine is located in the origin, two wing machines respectively lie in $\left(x_{i}, y_{i}\right),(\mathrm{i}=1,2,3)$. The baseline length of the host machine to the wing machines is $d_{i},(i=1,2)$, the distance between the target and host machine is $r_{0}$, and the distance between the target and wing machines is $r_{i}(i=1,2)$. Supposing that the arrangement of system stations and the position of targets as shown in Fig. 1.

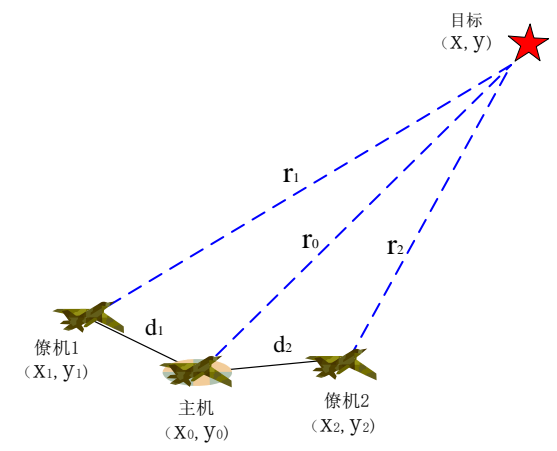

Fig.1. The schematic diagram of passive TDOA

The distance difference between radiation source to host machine and radiation source to wing machines is:

(1)

$$
\Delta r_{i}=r_{i}-r_{0}
$$

In this formula:

$$
r_{i}=\sqrt{\left(x-x_{i}\right)^{2}+\left(y-y_{i}\right)^{2}}(i=0,1,2)
$$

(2)

Where $\Delta r_{i}=c \cdot \Delta t_{i}, \Delta t_{i}$ is the measured value of time difference.

The matrix of linear equations established by (1):

(3)

$$
A X=B
$$

In this equation:

$$
A=\left(\begin{array}{ll}
x_{0}-x_{1} & y_{0}-y_{1} \\
x_{0}-x_{2} & y_{0}-y_{2}
\end{array}\right), X=\left(\begin{array}{l}
x \\
y
\end{array}\right), B=\left(\begin{array}{l}
k_{1}+\Delta r_{1} \cdot r_{0} \\
k_{2}+\Delta r_{2} \cdot r_{0}
\end{array}\right)
$$

(4)

Where $k_{i}=\left(\Delta r_{i}^{2}+x_{0}^{2}+y_{0}^{2}-x_{i}^{2}-y_{i}^{2}\right) / 2, \quad(i=1,2)$. 
Supposing $K=\left(\begin{array}{ll}k_{1} & k_{2}\end{array}\right), R=\left(\begin{array}{ll}\Delta r_{1} & \Delta r_{2}\end{array}\right), B=K+r_{0} \cdot R, C=A^{-1}$, so $C=\left(\begin{array}{ll}c_{11} & C_{12} \\ C_{21} & C_{22}\end{array}\right)=\left(\begin{array}{l}C_{1} \\ C_{2}\end{array}\right)$.

The result is:

$\left\{\begin{array}{l}x=C_{1} \cdot K+r_{0} \cdot C_{1} R \\ \mathrm{y}=C_{2} \cdot K+r_{0} \cdot C_{2} R\end{array}\right.$

(5)

In this formula:

$$
\left\{\begin{array}{l}
r_{0}=\left(-b \pm \sqrt{b^{2}-a c}\right) / a \\
a=\left(C_{1} R\right)^{2}+\left(C_{2} R\right)^{2}-1 \\
b=\left(C_{1} K-x_{0}\right) C_{1} R+\left(C_{2} K-x_{0}\right) C_{2} R \\
c=\left(C_{1} K-x_{0}\right)^{2}+\left(C_{2} K-x_{0}\right)^{2}
\end{array}\right.
$$

(6)

There are two values in (6), we can use the direction finding information of one wing machine to remove a false target information.

Now we analyze the location accuracy of TDOA.

On both ends of the differential location equation, you can get:

$$
d X=C^{-1}\left(d R-d X_{s}\right)
$$

(7)

In this equation:

$$
\begin{aligned}
& d X=\left(\begin{array}{ll}
d x & d y
\end{array}\right)^{\mathrm{T}}, \quad C=\left(\begin{array}{lll}
\frac{\left(x-x_{1}\right)}{r_{1}}-\frac{\left(x-x_{0}\right)}{r_{0}} & \frac{\left(y-y_{1}\right)}{r_{1}}-\frac{\left(y-y_{0}\right)}{r_{0}} \\
\frac{\left(x-x_{2}\right)}{r_{2}}-\frac{\left(x-x_{0}\right)}{r_{0}} & \frac{\left(y-y_{2}\right)}{r_{2}}-\frac{\left(y-y_{0}\right)}{r_{0}}
\end{array}\right), d R=\left(\begin{array}{ll}
d \Delta r_{1} & \left.d \Delta r_{2}\right)^{\mathrm{T}},
\end{array}\right) \\
& d X_{s}=\left(\begin{array}{ll}
k_{1}-k_{0} & k_{2}-k_{0}
\end{array}\right)^{\mathrm{T}}, \quad k_{0}=\frac{\left(x-x_{0}\right)}{r_{0}} d x_{0}+\frac{\left(y-y_{0}\right)}{r_{0}} d y_{0}, k_{i}=-\frac{\left(x-x_{i}\right)}{r_{i}} d x_{i}-\frac{\left(y-y_{i}\right)}{r_{i}} d y_{i} \text { 。 } \\
& \text { If } B=C^{-1}, \text { you can get: } \\
& d X=B\left(d R-d X_{s}\right)
\end{aligned}
$$

(8)

Because the time difference measurement all contain the error of arrival time measured by the host machine, so the measurement error of every $\Delta r_{i}$ is relevant. Supposing that the measurement error is zero-mean after correcting by system, and every position error is independent, thus the covariance matrix of location error is:

(9)

$$
\begin{aligned}
P_{d X} & =E\left[d X \cdot d X^{T}\right]=B\left\{E\left[d R \cdot d R^{T}\right]+E\left[d X_{s} \cdot d X_{s}{ }^{T}\right]\right\} B^{T} \\
& =B\left(\begin{array}{cc}
\sigma^{2}{ }_{\Delta r 1}+2 \sigma^{2}{ }_{s} & \eta_{12} \sigma_{\Delta r 1} \sigma_{\Delta r 2}+\sigma^{2}{ }_{s} \\
\eta_{12} \sigma_{\Delta r 1} \sigma_{\Delta r 2}+\sigma_{s}^{2} & \sigma^{2}{ }_{\Delta r 2}+2 \sigma_{s}^{2}
\end{array}\right) B^{T}
\end{aligned}
$$

Where $\sigma_{\Delta r i}^{2}$ is the standard deviation of the measurement error between host machine and the ith wing machine, $\eta_{i j}$ is the correlation coefficient between $\Delta r_{i}$ and $\Delta r_{j}, \sigma_{s}^{2}$ is position error.

When the error follow normal distribution, the error area is a ellipse, which is called elliptical error probable(EEP). The error ellipse can limit the estimated value of radiation source position clearly. EEP is used to measure the location performance in the paper.

In allusion to remote radiation source targets, when the distance between host machine and wing machines is short, the reliability of location result is poor. For example, we assume that the target is away from host machine $150 \mathrm{~km}$, and the two wing machines are both away from host machine $20 \mathrm{~km}$, at the same time, host machine and wing machines is linear array. Host machine is located at 
$(0 \mathrm{~km}, 0 \mathrm{~km})$, and the two wing machines are located at $(-14 \mathrm{~km}, 14 \mathrm{~km})$ and $(14 \mathrm{~km},-14 \mathrm{~km})$ respectively. The target is located at $(75.0 \mathrm{~km}, 129.9 \mathrm{~km})$. When the root-mean-square value of time difference measurement error is 35ns, 65ns and 95ns respectively, the position error ellipse as shown in Fig. 2.

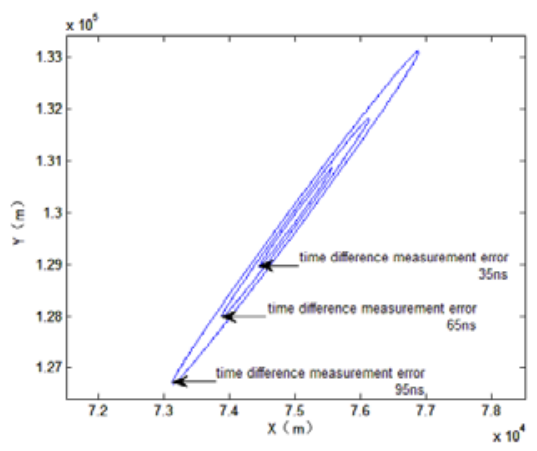

Fig.2. The schematic diagram of TDOA position error ellipse

Fig. 2 shows the position error ellipse of multiple passive station with short distance is centred by the true position of target, which is a long and thin ellipse with target direction as semi-major axis. When the root-mean-square value of time difference measurement accuracy is 95ns, the root-mean-square value of tangential accuracy is $98.7 \mathrm{~m}$.

If the distance between host machine and two wing machines is nearer, the EEP will longer and thiner. In order to promote the location accuracy, we can add the distance between host machine and two wing machines suitably. But the augment of distance will bring other problems.

In general, multi-machine synergetic passive location of remote radiation source have high tangential accuracy with low radial accuracy.

\section{Single pulse active location}

Active location detect the platform target position by launching detection pulse. Supposing that the direction of emission signals is $\theta$, and the target distance measured by pulse is $r$. Thus the relationship between the target position and distance and direction angle is:

$$
\left\{\begin{array}{l}
x=r \cos \theta=f_{x}(r, \theta) \\
y=r \sin \theta=f_{y}(r, \theta)
\end{array}\right.
$$

Supposing that the error between distance and direction angle is $\delta_{z}=\left[\delta_{r}, \delta_{\theta}\right]^{\mathrm{T}}$, the covariance matrix of the error is:

$$
\boldsymbol{P}_{z}=E\left[\delta_{z} \cdot \delta_{z}^{\mathrm{T}}\right]=\left[\begin{array}{cc}
\sigma_{r}^{2} & 0 \\
0 & \sigma_{\theta}^{2}
\end{array}\right]
$$

In allusion to position vector, we need work out the expression and covariance matrix of location error $\delta_{x}=\left[\delta_{x}, \delta_{y}\right]^{\mathrm{T}}$. The expression of $\delta_{x}$ is:

$$
\delta_{x}=\boldsymbol{A} \delta_{z}
$$

In the expression, the coefficient matrix is:

$$
\boldsymbol{A}=\left[\begin{array}{ll}
\frac{\partial f_{x}}{\partial r} & \frac{\partial f_{x}}{\partial \theta} \\
\frac{\partial f_{y}}{\partial r} & \frac{\partial f_{y}}{\partial \theta}
\end{array}\right]=\left[\begin{array}{cc}
\cos \theta & -r \sin \theta \\
\sin \theta & r \cos \theta
\end{array}\right]
$$

So the covariance matrix of platform target location error is: 


$$
\begin{aligned}
\boldsymbol{P}_{x} & =E\left[\delta_{x} \cdot \delta_{x}^{\mathrm{T}}\right] \\
& =\left[\begin{array}{cc}
\sigma_{x}^{2} & \rho_{x y} \sigma_{x} \sigma_{y} \\
\rho_{x y} \sigma_{x} \sigma_{y} & \sigma_{y}^{2}
\end{array}\right]
\end{aligned}
$$

(14)

Where:

$$
\left\{\begin{array}{l}
\sigma_{x}^{2}=\cos ^{2} \theta \cdot \sigma_{r}^{2}+r^{2} \sin ^{2} \theta \cdot \sigma_{\theta}^{2} \\
\sigma_{y}^{2}=\sin ^{2} \theta \cdot \sigma_{r}^{2}+r^{2} \cos ^{2} \theta \cdot \sigma_{\theta}^{2} \\
\rho_{x y} \sigma_{x} \sigma_{y}=\sin \theta \cos \theta \cdot \sigma_{r}^{2}-\sin \theta \cos \theta \cdot \sigma_{\theta}^{2}
\end{array}\right.
$$

In allusion to the active target detection under the condition of without angle tracking, especially under the condition of using instantaneous single-pulse measurement, the active location will acquire low tangential accuracy and high radial accuracy. For example, we assume that the target is away from host machine $150 \mathrm{~km}$, Host machine is located at $(0 \mathrm{~km}, 0 \mathrm{~km})$, and the target is located at $(75.0 \mathrm{~km}, 129.9 \mathrm{~km})$. When the root-mean-square value of distance error measured by host machine is $50 \mathrm{~m}, 100 \mathrm{~m}, 200 \mathrm{~m}$, the angle error is 0.5 degrees, 0.8 degrees and 1 degrees respectively. The position error ellipse as shown in Fig. 3.

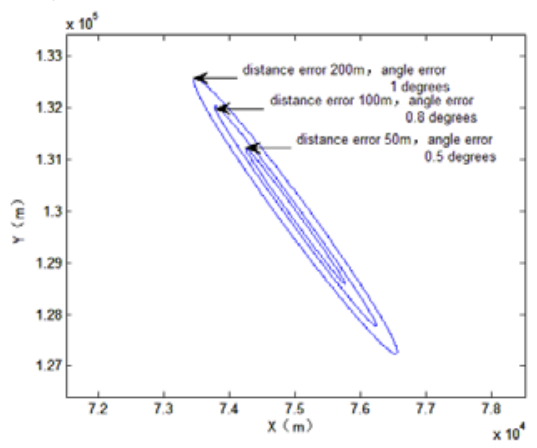

Fig.3. The schematic diagram of active detection position error ellipse

Fig. 3 shows the position error ellipse of single pulse active detection is centered by the true position of target, which is an ellipse with target direction as semi-major axis. In general, single pulse active location have high distance accuracy with low angle accuracy.

\section{Single pulse active location cooperate with passive location}

In this paper, the mode of single pulse active location cooperate with passive location is proposed, consisting of one host machine and two wing machines.

A. The workflow of the system

The system workflow single pulse active location cooperate with passive location is:

1. Synergetic passive location

Synergetic passive location of target radiation source is cooperated by host machine and two wing machines, passive location system have high tangential accuracy.

2. Single-pulse distance measurement of host machine

On the basis of the result of passive location, with the threat identification and reliability estimation, in allusion to the interested radiation source target, we can estimate the target is whether in the active detection range or not. And according to the direction obtained by passive location, launch single detection pulse to the direction, and acquire high distance location accuracy.

Because passive location is single-way propagation, which have the obvious advantage of find target earlier compared with active location. So the system work at the mode of passive location at first. It will launch detection pulse after estimating the target in the range of active detection. If it carry out valid distance measurement, it will achieve the combined location task of active and passive radar; if it can't fulfill valid detection, which means the target may be out of the range of active detection, it will launch a detection pulse to process active detection after a period of time. In 
this way, we can solve the distance matching question for active and passive detection.

3. Comprehensive location

We comprehensively analyze the result of single pulse active location at first, when the passive location and active location are both valid detection, after ensuring the radiation source platform of passive location and active location is the same platform, we carry out the calculation of comprehensive location.

B. The calculation of comprehensive location.

Supposing that the target position gained by multi-machine synergetic passive detection is:

$$
\hat{\boldsymbol{X}}_{1}=\boldsymbol{X}+\Delta \boldsymbol{X}_{1}
$$

The target position gained by single pulse active detection is:

$$
\hat{\boldsymbol{X}}_{2}=\boldsymbol{X}+\Delta \boldsymbol{X}_{2}
$$

Where the true position of target is $\boldsymbol{X}$, the estimated error is $\Delta \boldsymbol{X}_{1}, \Delta \boldsymbol{X}_{2}$. Supposing that $\hat{\boldsymbol{X}}_{1}$ and $\hat{\boldsymbol{X}}_{2}$ is in dependent, so the detection error follow zero-mean Gaussian distribution, $E\left\{\Delta \boldsymbol{X}_{1}\right\}=0, E\left\{\Delta \boldsymbol{X}_{2}\right\}=0$.

The covariance matrix of multi-machine synergetic passive detection is:

$$
\boldsymbol{P}_{1}=E\left\{\Delta \boldsymbol{X}_{1} \cdot \Delta \boldsymbol{X}_{1}^{\mathrm{T}}\right\}
$$

The covariance matrix of single pulse active detection is:

$$
\boldsymbol{P}_{2}=E\left\{\Delta \boldsymbol{X}_{2} \cdot \Delta \boldsymbol{X}_{2}^{\mathrm{T}}\right\}
$$

Using $\hat{\boldsymbol{X}}_{1}, \hat{\boldsymbol{X}}_{2}, \boldsymbol{P}_{1}, \boldsymbol{P}_{2}$, we can carry out the maximum likelihood estimation of target position:

$$
\hat{\boldsymbol{X}}=\left[\boldsymbol{P}_{1}^{-1}+\boldsymbol{P}_{2}^{-1}\right]^{-1}\left[\boldsymbol{P}_{1}^{-1} \hat{\boldsymbol{X}}_{1}+\boldsymbol{P}_{2}^{-1} \hat{\boldsymbol{X}}_{2}\right]
$$

Its covariance matrix of location error is:

$$
\begin{aligned}
\boldsymbol{P} & =E\left\{(\hat{\boldsymbol{X}}-\boldsymbol{X})(\hat{\boldsymbol{X}}-\boldsymbol{X})^{T}\right\} \\
& =\left[\boldsymbol{P}_{1}^{-1}+\boldsymbol{P}_{2}^{-1}\right]^{-1}
\end{aligned}
$$

Supposing that the root-mean-square value of multi-machine synergetic passive location time measurement is 95ns, the root-mean-square value of single pulse active location distance measurement is $200 \mathrm{~m}$, the root-mean-square value of angle error is 1 degrees. The position error ellipse of comprehensive location as shown in Fig. 4.

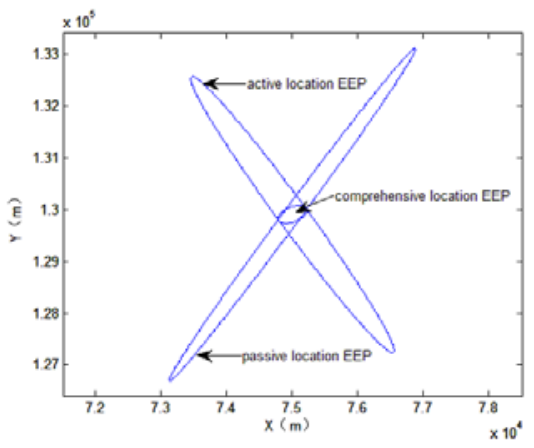

Fig.4. The schematic diagram of comprehensive detection position error ellipse

Fig. 4 shows single pulse active location cooperate with passive location can take advantage of the location performance complementary and improve the location accuracy. Even under the bad condition of the time measurement accuracy is $95 \mathrm{~ns}$, the distance measurement accuracy is $200 \mathrm{~m}$, 
we can still acquire high location accuracy with the root-mean-square value of $223.03 \mathrm{~m}$.

\section{Conclusion}

In this paper, the location performance of single pulse active location cooperate with passive location is analyzed. The remote location features and the error distribution features of multi-machine synergetic passive detection are analyzed in the issue at first. Then the location performance of single pulse active detection is proposed. In the end, the maximum likelihood estimation location result of the system is deduced as well as the location performance is analyzed. With the theoretical analysis and simulation approach proposed, under the condition of long distance, the multi-machine synergetic passive location can acquire high tangential location accuracy, single pulse active detection can acquire high radial location accuracy. If combining the location information of passive detection and single pulse active location, we can acquire higher location accuracy.

\section{References}

[1] He Ping. The principle of radar countermeasure[M]. Beijing: National Defend Industry Press, 2016.

[2] Zhang Kui, Luo Jingqing, Ma Xiantong. A method for multi-target information field location based on TDOA[J]. Journal of Detection \& Control, 2014, 36(4): 78-82.

[3] Oren Jean, Anthony J. Passive Localization and Synchronization Using Arbitrary Signals [J]. IEEE Transactions on Signal Processing, 2014, 62(8): 2143-2150.

[4] Luo Jingqing, Ma Xiantong, Wu Shilong. Location by Position Observing Information Tolerance Synthesize[J]. Electronics Optics \& Control, 2015, 22(4): 1-6.

[5] Li Hongwei, He Qingyi, Li Chun. The derivation and analysis of CRLB for moving multi-station passive TDOA[J]. Journal of Projectiles, Rockets, Missiles and Guidance, 2013, 33(6): 167-170.

[6] Cheneg Dongsheng, Li Xia, Wan Shanhu, Zhou Junwei. Analysis of the collocation and detection performance of the passive radar/radar combined detection system[J]. Modern Defense Technology, 36(5): 135-141.

[7] Zhang Zhengchao, Li Wenchen. Target location in integrative mode of radar location and passive location and its precise analysis[J]. Telecommunication Engineering, 2012, 52(11): 1758-1762.

[8] Cheng Dongsheng, Li Xia, Zhou Junwei. The comprehensive performance analysis of the radar/passive radar combined detection system[J]. Fire Control \& Command Control, 2009, 34(9): 95-98. 